\title{
EFFECTS OF MACROMIXING ON THE OREGONATOR MODEL OF THE BELOUSOV-ZHABOTINSKY REACTION IN A STIRRED REACTOR
}

\author{
T. J. HSU, C. Y. MOU and D. J. LEE ${ }^{\mathrm{b} *}$ \\ "Department of Chemistry, \\ 'Department of Chemical Engineering, National Taiwan University, Taipei, Taiwan, 106
}

(Received 16 May 1994; accepted for publication 12 September 1994)

\begin{abstract}
Macromixing effects on the oscillating Belousov-Zhabotinsky reaction based on the global fluid field have been investigated. The three-variable irreversible Oregonator and the networks-of-zones model with $2 \times N \times N$ zones were chosen for describing the chemical kinetics and the flow field, respectively. With high stirring rate, all zones in the tank will oscillate synchronously and the tank can be taken as an ideally well-mixing continuous-flow stirred-tank reactor. When stirring rate is decreased, locally simple oscillation patterns were found to be sustained, and the oscillation period was decreased accordingly. It is proposed that the output zone acts as an excitable nucleus which determines the overall oscillations. When stirring rate is reduced further, many local zones can be excited as nuclei, and apparent complex oscillations can result.
\end{abstract}

\section{INTROUUCTION}

It is well known that mixing may have a profound effect on selectivity or yield for certain chemical reactions (Levenspiel, 1972; Nauman and Buffham, 1983). A well-mixed continuous-flow stirred-tank reactor (CSTR) is in most cases only a limit which will never be attained in practice. However, various chemical kinetics have been studied in a stirred tank with the assumption that the medium was uniform. Such an assumption may introduce serious errors in systems with characteristic reaction time comparable to the mixing time [see Villermaux (1991) and the references cited therein].

The Belousov-Zhabotinsky (BZ) reaction, consisting of cerium-catalyzed oxidation of malonic acid by bromate in an acidic medium, is one of the best examples of a chemical system exhibiting both temporal and spatial instabilities far from equilibrium. It has been found experimentally in a stirred tank reactor, batchwise or continuous, that this reaction possesses complex dynamic behavior, such as periodic/aperiodic oscillation, hysteresis and chaos under certain circumstances [see Gyorgyi and Field (1992) and the references cited therein].

The first comprehensive mechanism for the BZ reaction was proposed by Field, Koros, and Noyes (FKN) in 1972. An abstract model derived from the FKN mechanism was proposed by Field and Noyes (1974), the so-called three-variable irreversible Oregonator (abbreviated as "Oregonator" in this work), which could qualitatively describe the oscillating behavior of the $\mathrm{BZ}$ reaction. The basic features of

"To whom correspondence should be addressed. the Oregonator have been further discussed by Tyson (1977, 1981), and DeKepper and Boissonade (1981). Showalter, Noyes and Bar-Eli (1978) refined the Oregonator to incorporate a reversible mechanism (SNB model). Lindberg et al. (1990) reported on the observation of large-scale chaos from a simulation with the SNB model. A well-mixed tank was assumed in all these studies.

In the last decade, however, much evidence has indicated that the stirring effects may affect the system dynamics both quantitatively and qualitatively (Roux et al., 1983; Menzinger et al., 1986; Menzinger and Giraudi, 1987; Dutt and Menzinger, 1990; Noszticzius et al., 1991; Orban and Epstein, 1992; Dutt and Muller, 1993; Ruoff, 1993; Vanag and Alfimov, 1993). Menzinger and Jankowski (1986) provided the first evidence of the existence of spatially distributed concentration fluctuations in a stirred tank. Hauser et al. (1992) performed experiments on minimal bromate oscillator in a stirred vessel with stirring rate up to $900 \mathrm{rpm}$, and concluded that such a high stirring rate was still far below the well-mixed limit. Menzinger et al. (1986) demonstrated that the way reactants had been fed would strongly affect the system dynamics even at a high stirring rate of $2500 \mathrm{rpm}$.

The importance of mixing is usually discussed in terms of the concepts of macromixing and micromixing. Macromixing treats the hydrodynamic aspect in the reactor, whilst micromixing deals with mixing processes with scales below the characteristic turbulence length (Levenspiel, 1972). Usually, the real condition of a reactor lies somewhere in between. The role of micromixing on complex chemical kinetics has been studied extensively (Horsthemke and Hannon, 1984; Hannon and Horsthemke, 1987; Puhl and 
Nicolis, 1986, 1987; Boissonade and DeKepper, 1987; Fox and Villermaux, 1990a, 1990b; Villermaux, 1991; Chang, 1992; David et al., 1992; Gyorgyi and Field, 1992; Rouff, 1993); however, only a few studies have paid attention to macromixing (Kumpinsky and Epstein, 1985; Luo and Epstein, 1986; Razon and Schmitz, 1987; Gyorgyi and Field, 1989; Ganapathisubramanian, 1991), none of which was based on the global fluid field character of a stirredtank reactor.

To search for a complete solution of the NavierStokes equations in a stirred tank is still very difficult. The networks-of-zones model, developed by Mann and co-workers, in which the reactor is divided into an assembly of backmixed zones with internal flow streams, provides a much simpler approach (Mann and Mavros, 1982, Knysh and Mann, 1984, Mann, 1986). This approach has been applied to modelling the fluid flow field in single-phase or multiple-phase stirred-tanks (Mann et al., 1987; Brucato and Rizzuti, 1988; Mann and Hackett, 1988; Voit and Mersmann, 1988; Mann and El-Hamuu<, 1991; Nienuw et al., 1992; Wang and Mann, 1992). Large lateral concentration variations were observed from these experiments and calculations. It was shown that such a model could represent well the global fluid field in a stirred tank (Mann et al., 1987).

The effect of macromixing has been investigated by two-compartment models (Kumpinsky and Epstein, 1985; Luo and Epstein, 1986; Gyorgyi and Field, 1989; Ganapathisubramanian, 1991), threecompartment models (Villermaux, 1991), or at most, four-compartment models (Hauser et al., 1992). Ganapathisubramanian (1991) noted that in a stirred tank, there could exist many small zones containing different concentrations. Since the concentration fluctuations and the sophisticated interactions between various parts of the tank should be highly dependent on the turbulent fluid flow field, a discussion based on a more realistic global fluid field in a stirred tank was desirable.

In this paper, a first attempt at investigating the macromixing effects on the oscillating $\mathrm{BZ}$ reaction based on a global fluid field model (networks-of-zones model with $2 \times N \times N$ zones) is presented. For simplicity, the Oregonator is chosen to describe the chemical kinetics. Following a brief discussion on the Oregonator and the flow model, the effects of stirring on the concentration field, the oscillation periods, and the apparent complex oscillations are presented. Finally, a possible explanation of the observed stirring effects on the $B Z$ reaction is proposed.

\section{THE OREGONATOR}

The Oregonator is a kinetic model, suggested by Field and Noyes (1974) as a model of the FKN mechanism, as follows:

$$
\begin{aligned}
& A+y \stackrel{K_{1}}{\longrightarrow} x+P, \\
& x+y \stackrel{K_{2}}{\longrightarrow} P+P,
\end{aligned}
$$

$$
\begin{gathered}
A+x \stackrel{K_{3}}{\longrightarrow} 2 x+z, \\
x+x \stackrel{K_{4}}{\longrightarrow} A+P, \\
z \stackrel{K_{3}}{\longrightarrow} f y,
\end{gathered}
$$

where $A=\left[\mathrm{BrO}_{3}^{-}\right], P=[\mathrm{HOBr}], x=\left[\mathrm{HBrO}_{2}\right], y=$ $\left[\mathrm{Br}^{-}\right]$, and $z=2\left[\mathrm{Ce}^{4+}\right]$. The concentration of $A$ is taken as constant. $K_{1}$ to $K_{5}$ are rate constants, and $f$ is a noninteger stoichiometric parameter (DeKepper and Boissonade, 1981).

The differential equations based on kinetic model eqs (1a)-(le) are as follows:

$$
\begin{aligned}
\frac{\mathrm{d} x}{\mathrm{~d} t_{1}} & =K_{1} A y-K_{2} x y+K_{3} A x-2 K_{4} x^{2}, \\
\frac{\mathrm{d} y}{\mathrm{~d} t_{1}} & =-K_{1} A y-K_{2} x y+f K_{5} z \\
\frac{\mathrm{d} z}{\mathrm{~d} t_{1}} & =K_{3} A x-K_{5} z .
\end{aligned}
$$

The dimensionless groups are defined as follows:

$$
\begin{aligned}
X & =\frac{K_{2}}{K_{1} A} x, \\
Y & =\frac{K_{2}}{K_{3} A} y, \\
Z & =\frac{K_{2} K_{5}}{K_{1} K_{3} A^{2}} z, \\
t & =\sqrt{K_{1} K_{3}} A t_{1}, \\
s & =\sqrt{\frac{K_{3}}{K_{1}}} \\
\omega & =\frac{K_{5}}{A \sqrt{K_{1} K_{3}}}, \\
q & =\frac{2 K_{1} K_{4}}{K_{2} K_{3}} .
\end{aligned}
$$

With the terms in eqs $(3 \mathrm{a})-(3 \mathrm{~g})$, the dimensionless forms for the three components in the Oregonator in a perfect CSTR can be written as follows:

$$
\begin{aligned}
\frac{\mathrm{d} X}{\mathrm{~d} t} & =s\left(Y-X Y+X-q X^{2}\right)-\frac{1}{\tau}\left(X-X_{0}\right), \\
\frac{\mathrm{d} Y}{\mathrm{~d} t} & =(-Y-X Y+f Z) / s-\frac{1}{\tau}\left(Y-Y_{0}\right), \\
\frac{\mathrm{d} Z}{\mathrm{~d} t} & =w(X-Z)-\frac{1}{\tau}\left(Z-Z_{0}\right) .
\end{aligned}
$$

With the rate constants and parameters given in Field and Noyes (1974), and $\left[\mathrm{HBrO}_{2}\right]=5.025 \times$ $10^{-11} \mathrm{X},\left[\mathrm{Br}^{-1}\right]=3.000 \times 10^{-7} \mathrm{Y},\left[\mathrm{Ce}^{+4}\right]=2.412 \times$ $10^{-8} Z, s=77.27, q=8.375 \times 10^{-6}$, and $w=0.161 K_{5}$. Equations (4a)-(4c) hence contain three parameters, 

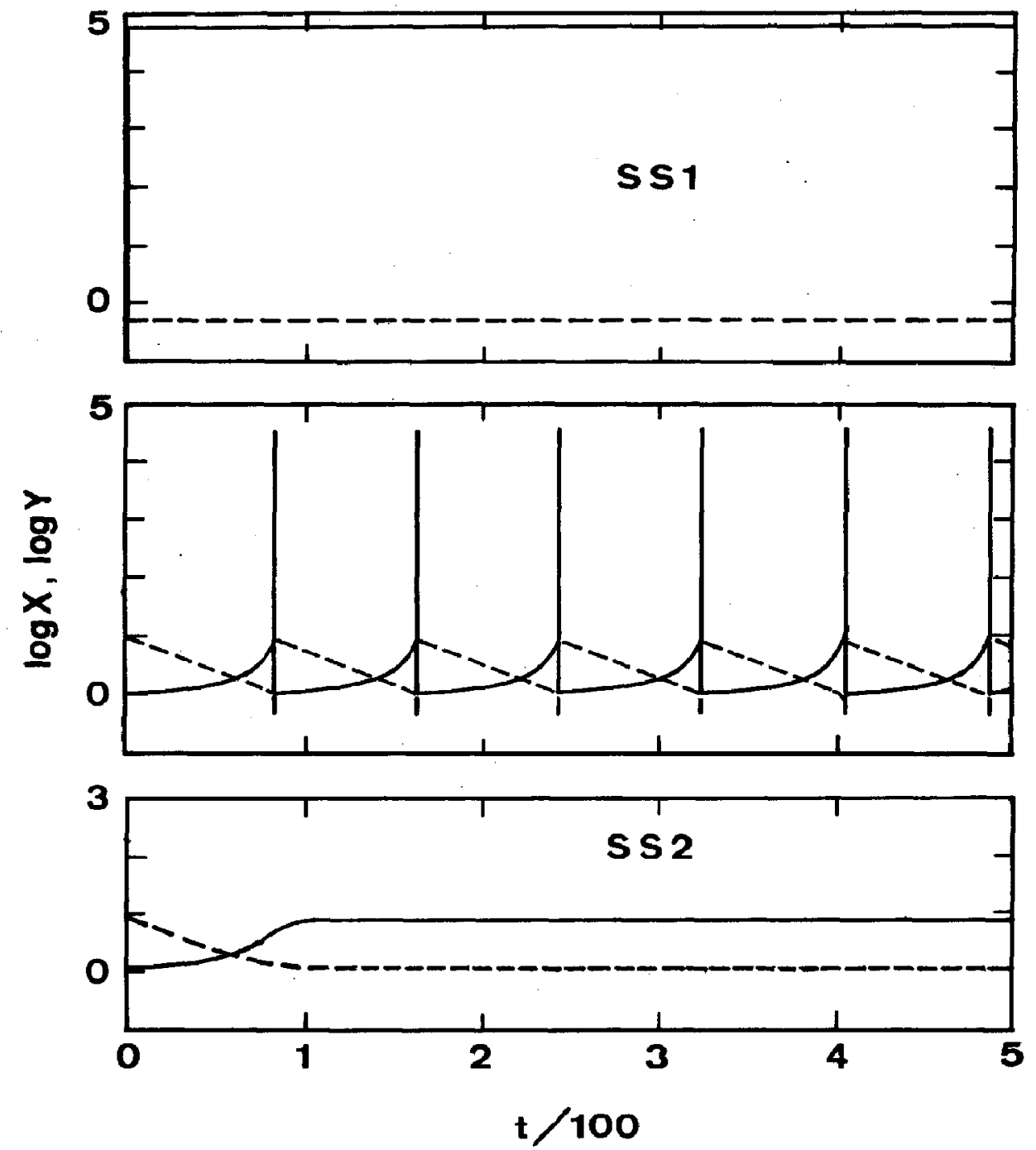

Fig. 1. Concentration of $X$ (solid lines) and $Y$ (dashed lines) vs time plot in a closed, perfectly mixed reactor. $K_{5}-200$. Initial conditions all set as 0.01 . (a) $f=0.5$, (b) $f=0.9$, and (c) $f=1.3$.

$f$ and $K_{5}$ for chemical kinetics, and $\tau$ the residence time for the feed flow rate. Since eq. (4) is a highly nonlinear ODE system, Gear's method was employed as the integration tool with the maximum relative error of $10^{-6}$.

\section{Closed system}

In a closed system, the residence time becomes infinite and eqs (4a)-(4c) reduced to the ones proposed in Field and Noyes (1974). Take the calculation with $K_{5}=200$ as an example. Equations (4a)-(4c) were solved numerically under no-flow conditions, and the system dynamies are shown in Fig. 1(a)-(c) for $f=0.5,0.9$, and 1.3 , respectively.

When $f=0.5$, the system attains a steady state denoted as "SS1" where $X$ and $Z$ are high. When $f$ is increased to 1.3 , the system shifts to another steady state with low $X$ and $Z$ values, denoted as "SS2". In between, there exists an oscillation region. An oscillation period of 80.4 for $f=0.9$ is observed, as shown in Fig. 1(b). A stability diagram characterizing the oscillatory and steady-state regimes in a closed system has been given in Field and Noyes (1974). The initial conditions, though having no effects on the oscillation period, have a strong effect on the initial induction time.

\section{Flow system}

In a continuous-flow system, two more parameters are added: the residence time and the feed concentrations. The oscillation period is insensitive to the feed concentration, but it is strongly affected by the feed flow rate. Again, take the case with $K_{5}=200$ as an example. Figure 2 demonstrates the variation of 


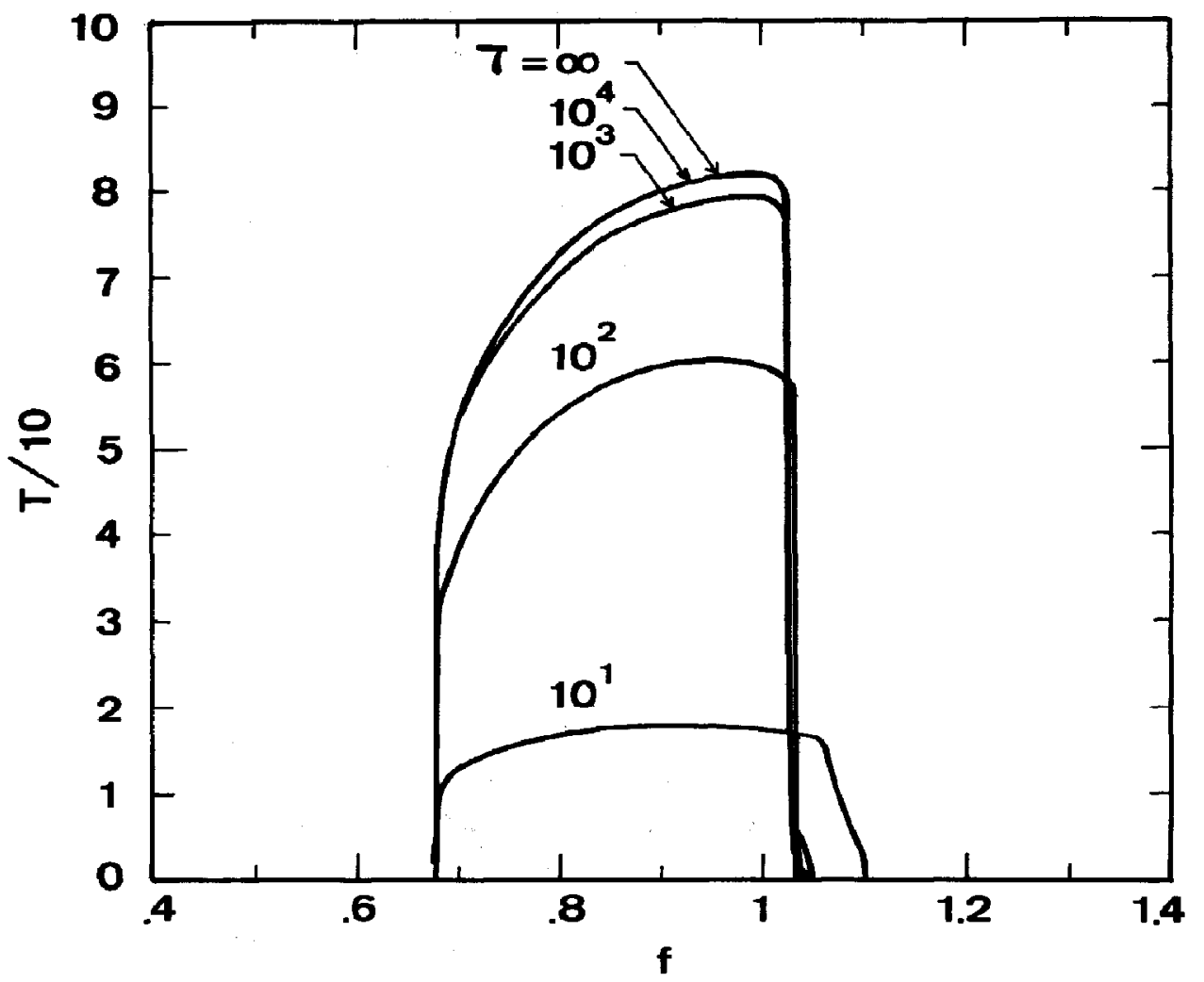

Fig. 2. $T$ vs $f$ plot for an ideal CSTR. $K_{5}=200$. Outside the oscillatory region $T$ became infinite.

oscillation period $T$ against $f$, with residence time $\tau$ as a parameter. $T$ decreases with increasing feed flow rate, and the $T$ vs $f$ curve exhibits a local maximum when $f$ is near $0.9-1.0$.

A stability diagram similar to that proposed in Field and Noyes (1974) is shown in Fig. 3 with $\tau=1000$. The situations with other $\tau$ values are all alike and hence are not repeated here. At a fixed $\tau$, there exists a critical $K_{5}$ value above which no oscillation occurs. From Figs 2 and 3, a state with $\left(K_{5}, f\right)=(200,0.9)$, which is in the middle of the simple relaxation oscillation region, is chosen for further discussions.

The oscillation period decreases with increasing feed flow rate, as demonstrated in Fig. 4. These data are taken as standards for latter comparisons. When $Q_{F}$ is small, the oscillation period can be approximated as a linear function of the feed flow rate.

\section{THE NETWORKS OF ZONES MODEL}

The flow system of the networks-of-zones model is basically the same as that employed in Knysh and Mann (1984). The model comprises $2 \times N \times N$ spatial zones. Each zone is perfectly mixing and has a through-flow of flow rate $Q_{k}$, and an exchange flow with the nearest adjacent zones due to turbulence fluctuation of flow rate $\alpha Q_{i}$. The configuration for the model with $N=10$ is shown schematically in Fig. 5 . The relation between $\alpha$ and stirring velocity has been discussed in Mann et al. (1987). In this study, the zone in the middle of the tank $[(5,10)$ for the $2 \times 10 \times 10$ model $]$ and that at the top-left $[(1,20)$ for the $2 \times 10 \times 10$ model] were selected as the input and output zone, respectively. The volume for each zone was set as unity.

Mass balance equations can be written for each zone. For the configurations shown in Fig. 5, for example, the following mass balance equation for zone $(5,2)$ can be written for component $I$ :

$$
\begin{aligned}
\frac{\mathrm{d} C_{I_{5,2}}}{\mathrm{~d} t} & =\frac{Q_{i}}{V_{5,2}}\left[C_{I_{6,2}}+\alpha\left(C_{I_{5,1}}+C_{I_{5,3}}\right)\right. \\
& \left.-(1+2 \alpha) C_{I_{5,2}}\right]+r_{I}
\end{aligned}
$$

where $I$ stands for $X, Y$, or $Z ; V_{5,2}=1.0$; and $r_{I}$ values are the reaction terms. For the zone into which the feed entered, the mass balance equation was modified according to Mann and El-Hamouz (1991).

In a no-reaction study, a set of $2 \times N \times N$ ODEs was solved. When the Oregonator was substituted into $r_{I}$ values, $6 \times N \times N$ stiff ODEs were to be solved simultaneously. In this study, the number of ODEs ranged from $600(N=10)$ to $5400(N=30)$.

In order to keep the global flow pattern in a stirred tank independent of $N$, the values of $Q_{i}$ and $Q_{F}$ should 
Effects of macromixing on the Belousov-Zhabotinsky reaction

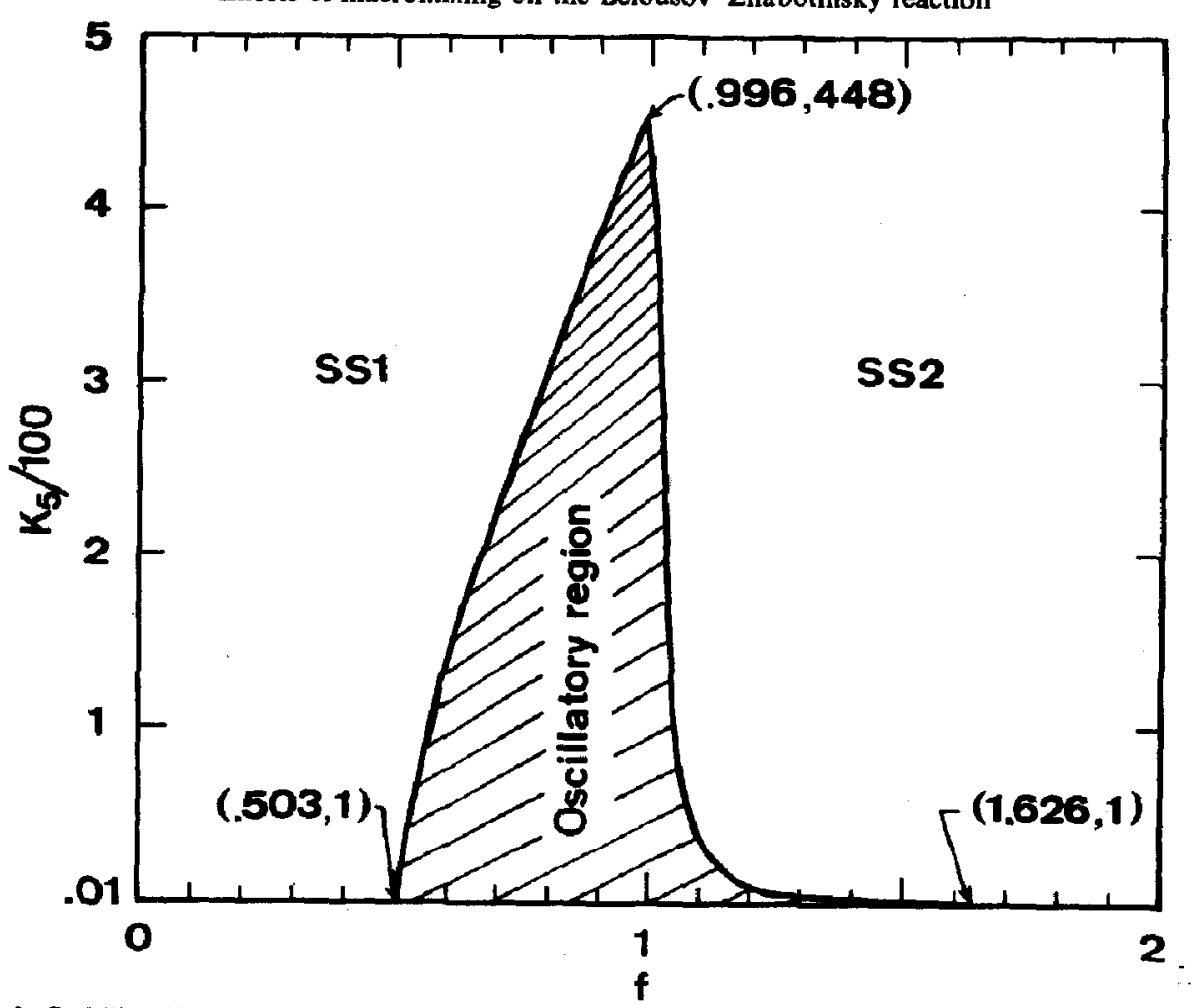

Fig. 3. Stability diagram for the steady states and oscillatory regions for an ideal CSTR with $\tau=1000$.

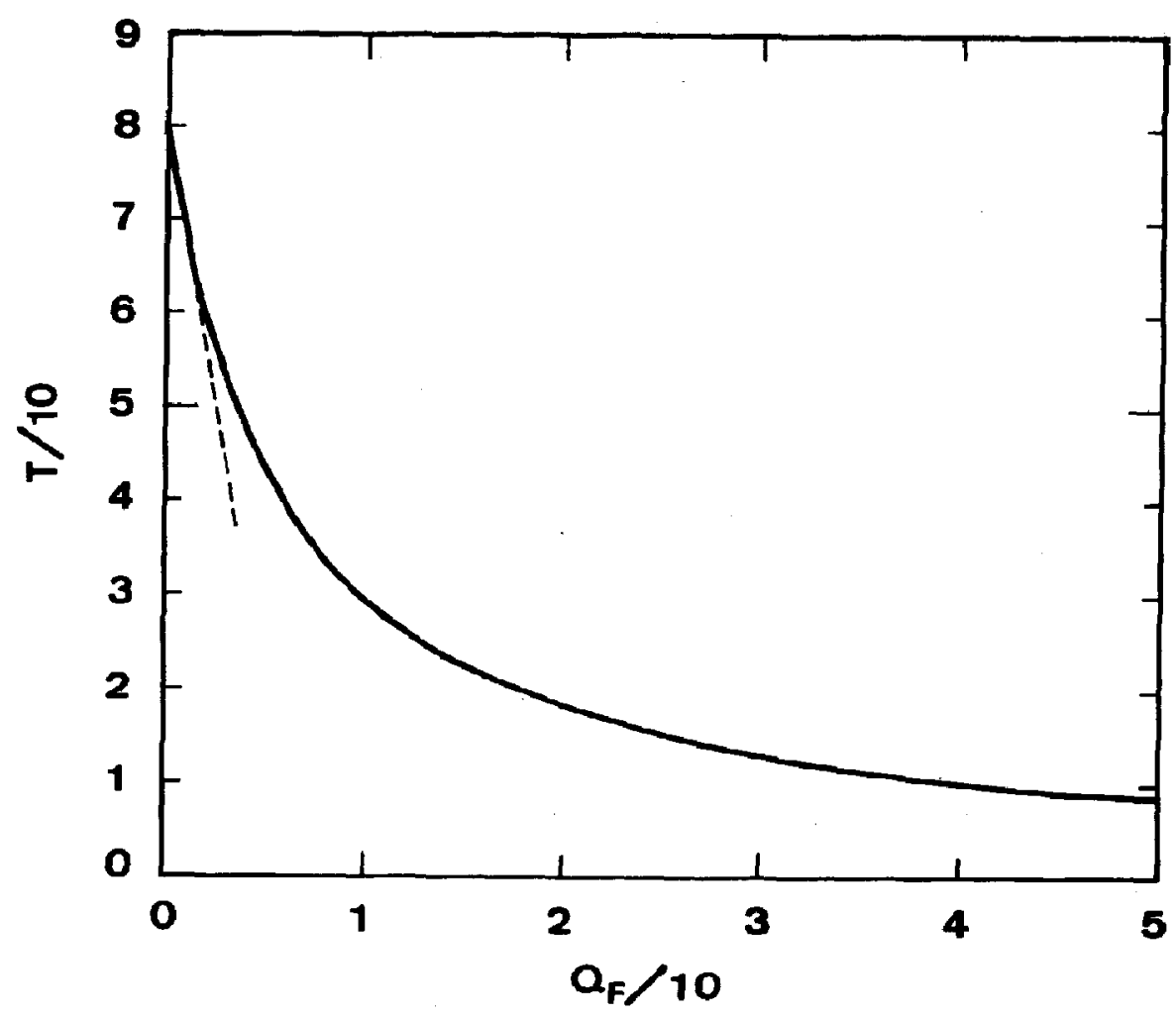

Fig. 4. $T$ vs $Q_{F}$ plot for an ideal CSTR. $K_{s}=200, f=0.9$. 


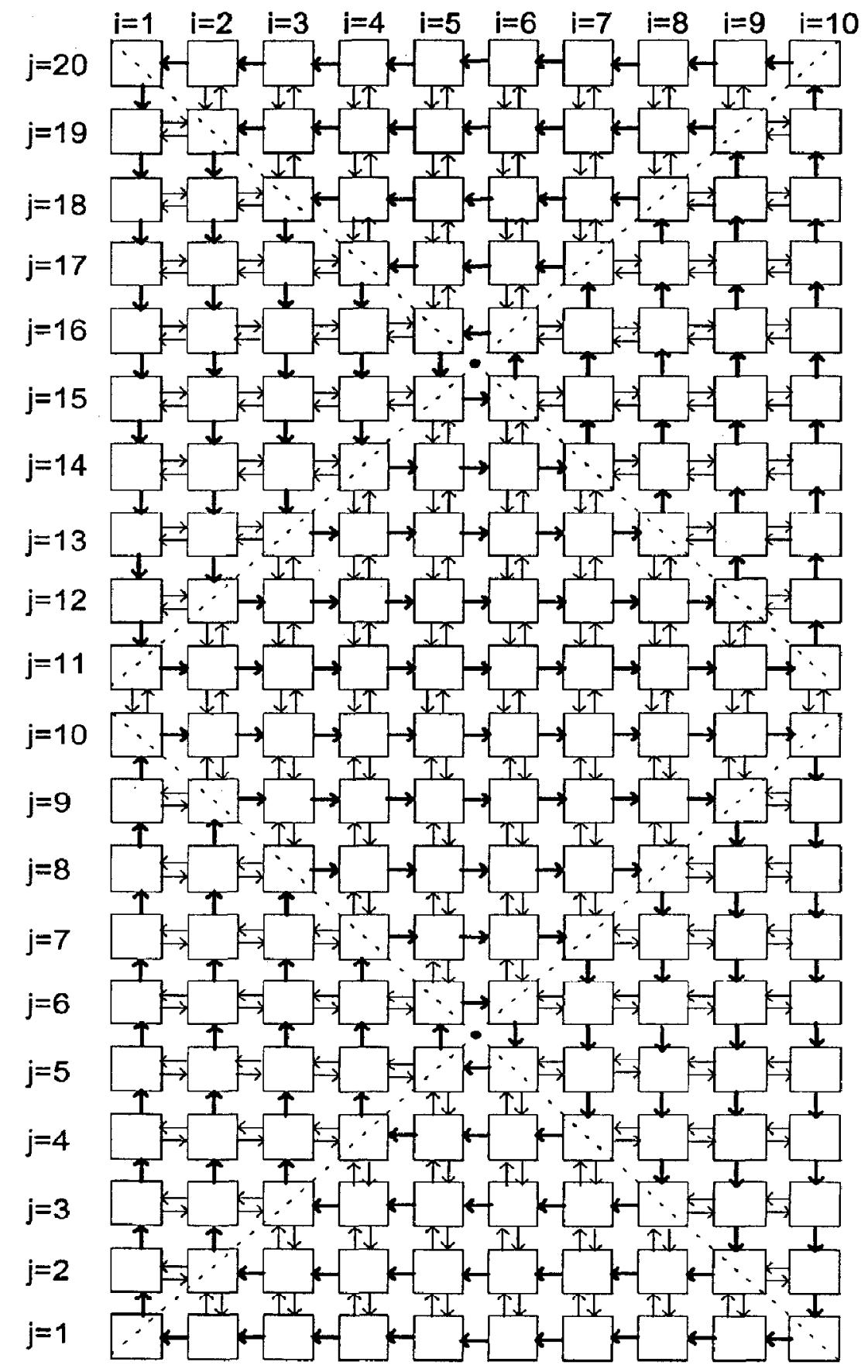

Fig. 5. A $2 \times 10 \times 10$ networks-of-zones model. Zones $(5,10)$ and $(1,1)$ are the input and output port, respectively. The stirrer is located between zone $(1,10)$ and $(1,11)$.

scale with $N$; that is, they become $2 Q_{i}$ and $4 Q_{F}$ when $N$ is doubled.

Some simulations were conducted assuming a step-change input of a tracer with the parameter sets shown in Table 1 . When $Q_{i} / Q_{F}$ is greater than about 2 , the calculated output tracer curve is independent of $N$ for $N$ ranges of 10-30. In the following calculations, therefore, $Q_{i}$ was kept greater than $2 Q_{F}$, and $N$ was taken as 10 if not otherwise mentioned.

\section{MIXING EFFECTS ON THE BZ REACTION}

\section{Simple oscillations}

When the internal flow rate is high, no severe lateral heterogeneity is observed except at the beginning of 
Table 1. The parameter sets used in this work

\begin{tabular}{rccl}
\hline No. & $N$ & $Q_{i}$ & \multicolumn{1}{c}{$Q_{F}$} \\
\hline 1 & 10 & 50 & 0.2 \\
2 & 10 & 25 & 0.2 \\
3 & 10 & 10 & 0.2 \\
4 & 10 & 5 & 0.2 \\
5 & 10 & 1 & 0.2 \\
6 & 10 & 0.5 & 0.2 \\
7 & 20 & 10 & 0.8 \\
8 & 30 & 15 & 1.8 \\
9 & 10 & 50 & 0.002 \\
10 & 10 & 10 & 0.002 \\
11 & 10 & 5 & 0.002 \\
12 & 10 & 1 & 0.002 \\
13 & 10 & 0.5 & 0.002 \\
14 & 10 & 0.1 & 0.002 \\
15 & 20 & 100 & 0.8 \\
16 & 30 & 150 & 1.8 \\
\hline
\end{tabular}

the simulation. Oscillations in all zones are almost synchronous.

The majority of experiments were performed with macroprobes (an electrode or a UV sensor) for monitoring the concentration variation in the stirred tank (Argoul et al., 1987; Sevcik and Adamcikova, 1988, 1989; Dutt and Menzinger, 1990; Ochiai and Menzinger, 1990; Hauser et al., 1992). However, some workers employed a microelectrode (Menzinger and Jankowski, 1986, 1990; Menzinger and Dutt, 1990; Dutt and Menzinger, 1992). Here the concentration variation for zone $(5,2)$ was taken as the response of the microprobe, and the average concentration over zone $(5,2)$ and the four nearest neighbors $(5,1),(5,3)$. $(4,2)$ and $(6,2)$, was the response of a macroprobe.

The calculated local and the average concentration profiles are shown in Fig. 6(a) and (b). The dynamics are consistent with the results for a perfect CSTR [Fig. 1(b)]. The phase portrait is just a limit cycle for the Oregonator. Clearly, under such a circumstance, the stirred tank can be taken as a perfect CSTR. No obvious difference was observed with either a microelectrode or a macroelectrode in such a case.

When $Q_{i}$ is decreased gradually, the simple oscillation pattern for all zones in the tank can still be maintained locally, with a time lag depending on the zone position. An oscillation is always ignited from the output zone, and then the high concentration wave front moves, following the flow field through the upper and the lower major loops, respectively, according to action of the stirrer.

To demonstrate possible interactions between nearby zones, Fig. 7 shows the cross-correlation coefficients for component $\boldsymbol{X}$ between zone $(5,2)$ with, respectively, the four nearest neighbors. Perfect synchronism was found for all five coefficients when stirring rate was high [Fig. 7(a)]. When stirring rate was decreased, the position of maximum correlation shifted with a finite time delay [Fig. 7(b)]. Note that the time lags of all zones in an oscillation cycle were generally less than 1 , which were much less than the oscillation period of $60-80$, or the system residence time of 1000 . Therefore, the oscillation periods recorded in all individual zones were approximately the same. The region where simple oscillation pattern was sustained in the vessel, though with finite time lags, is referred to as the "SO" region.

In the "SO" region, both the local and average oscillation periods are affected by the stirring rate. Figure 8 is a plot for the oscillation period vs the internal flow rate with two residence times (Parameter set nos 1-6 and 9-14). Up to $21 \%$ reduction of oscillation period can be found for $Q_{F}=0.2$ when $Q_{i}$ is decreased from 50 to 1 . This is qualitatively consistent with experimental findings (Menzinger and Jankowski, 1986, 1990; Lopez-Tomas and Sagues, 1991). With the Oregonator as the chemical kinetics system, this is the first time that the reduction of the oscillation period with increasing stirring rate was simulated by a hydrodynamic method.

When $Q_{F}$ is reduced to 0.002 , the oscillation period is not affected by the internal flow rate, even when $Q_{i}$ is as low as 0.1 .

To check whether the calculated results are independent of the flow model, two sets of simulations with different residence times (Parameter set nos 4, 7, 8 and $1,15,16)$ were performed. The pattern and period of oscillations were found to be almost $N$-independent for $N=10-30$.

\section{Complex oscillations}

When internal flow rate is decreased further, complex oscillations occur. The calculated global concentration field for Parameter set no. 6 is demonstrated in Fig. 9. Several high-concentration wave fronts may coexist in the vessel, and large lateral heterogeneity exists all over the tank. The region where complex oscillations occur is referred to as the "CO" region. Again, such apparent complex dynamics are generated in a hydrodynamic way.

The average and the local concentrations are shown in Fig. 10(a) and (b), respectively. Although with differences in details, the major characteristics for the two time sequences are similar, which is also consistent with the experimental results by Menzinger and Jankowski (1986).

Cross-correlation coefficients for low internal flow rate are shown in Fig. 11; a local synchronism is found to be held approximately between the neighboring zones. However, the correlation soon becomes very weak when the two zones are far apart.

\section{DISCUSSIONS}

Lengthening of oscillation period

A possible mechanism for the lengthening of the oscillation period under stirring is discussed in this section.

In the FKN model, the key substance in the $\mathrm{BZ}$ reaction is the bromide ion, which switches the reactions between two processes (Field et al., 1972). When $\left[\mathrm{Br}^{-}\right]<\left[\mathrm{Br}^{-}\right]_{\text {crit }}$, the bromide ion is consumed to form $\mathrm{Br}_{2}$ (reaction " $A$ "), which further reacts 

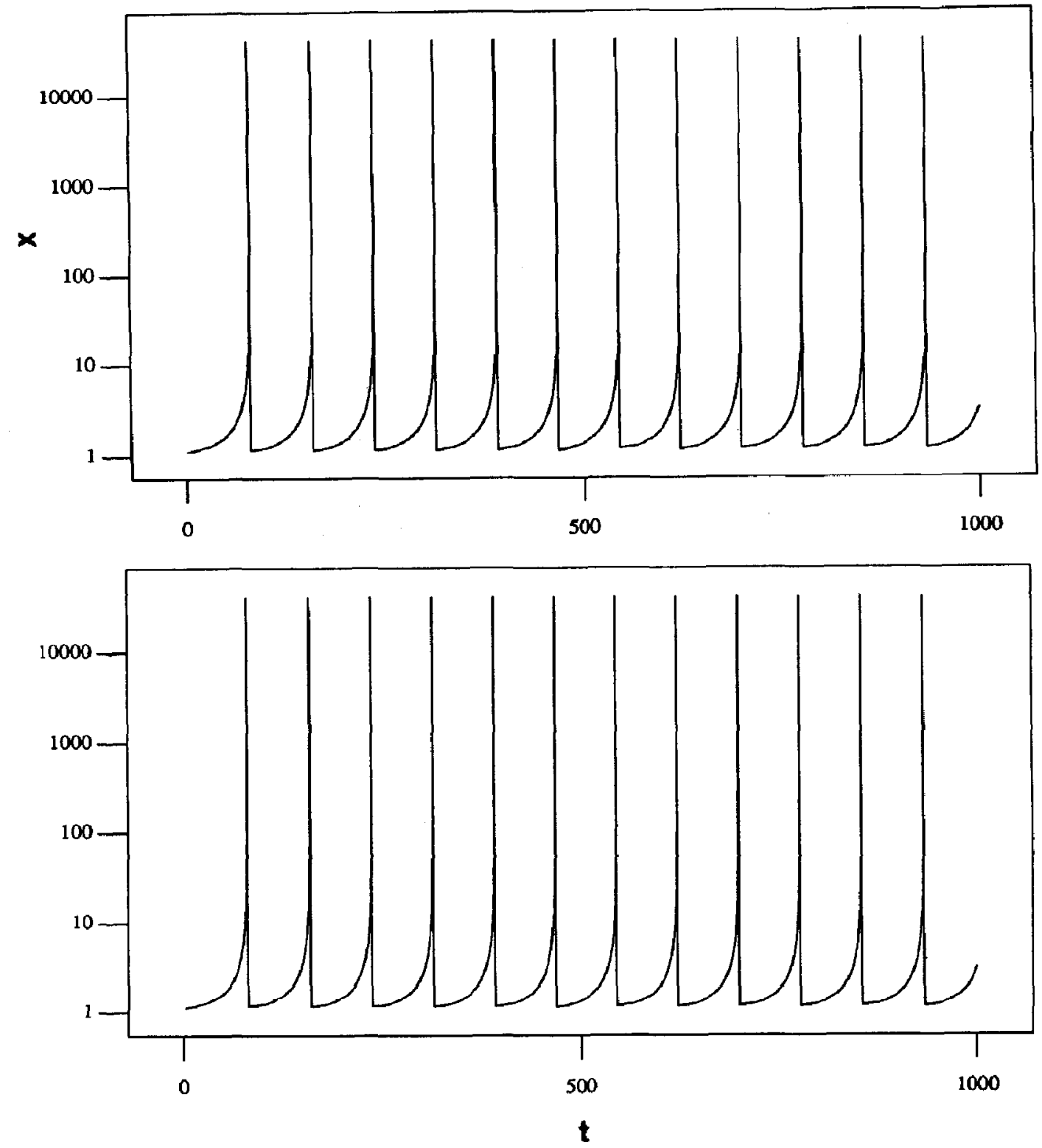

Fig. 6. Concentration for component $X$ vs time. $K_{5}=200, f=0.9$, Parameter set no. $1, \tau=1000$. (a) Local concentration for zone $(5,2)$, and (b) average concentration for zone $(5,2)$ and its four nearest neighbors.

with the organic substance. Due to such a process, the concentration of the bromide ion will then be reduced subsequently until $\left[\mathrm{Br}^{-}\right]>\left[\mathrm{Br}^{-}\right]_{\text {crit }}$, where an autocatalytic reaction is triggered to form $\mathrm{HBrO}_{2}$, with a metal ion as a catalyst. Based on this basic model, at least four proposals have been made to explain the stirring effect on the oscillation period.

The first was the enhancement of oxygen transfer across the liquid/gas interface by stirring, which would cause more bromide ion to form, which would lengthen the time required for $\left[\mathrm{Br}^{-}\right]$to drop below the critical value, and also prolong the oscillation period. If the oxygen transfer is too efficient, the oscillation may be killed altogether (Sevcik and Adamcikova, 1988).

Later experiments showed that the stirring effects still existed even in an inert atmosphere (Sevick and Adamcikova, 1988, 1989). The enhancement of $\mathrm{Br}_{2}$ removal due to stirring, which would slow down the production of bromomalonic acid, was proposed to be responsible for the increase of oscillation period (Lopez-Tomas and Sagues, 1991; Pojman et al., 1992). Similar effects could be observed when $\mathrm{Br}_{2}$ was adsorbed onto the vessel wall or the electrode, or when 

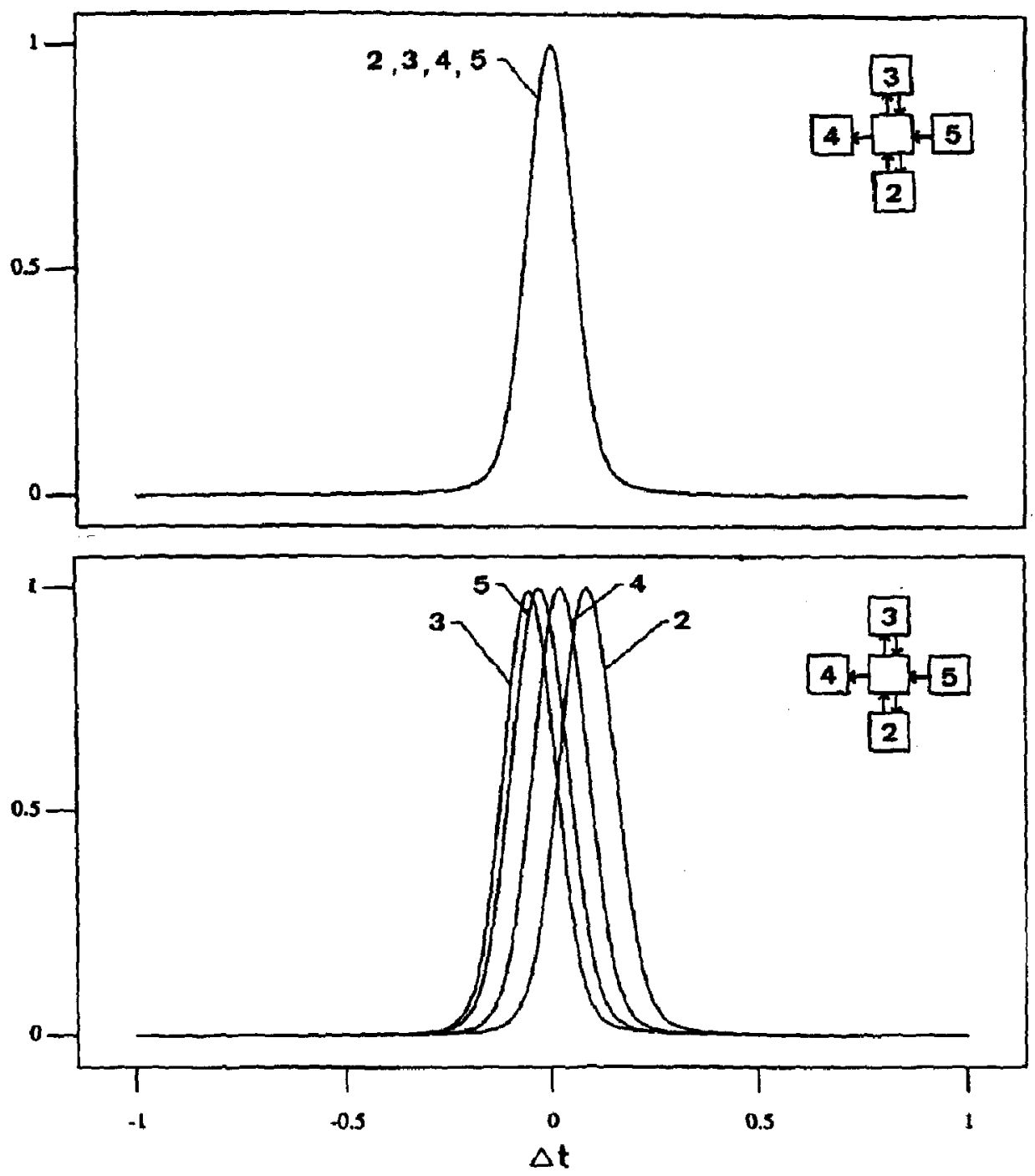

Fig. 7. Crass-correlation coefficients between zone $(5,2)$ with its four neighbors $(2-5)$. (a) High stirring rate (Parameter set no. 1), and (b) medium stirring rate (Parameter set no. 4).

nitrogen purging gas was used (Menzinger and Jankowski, 1990).

Noszticzius et al. (1991) proposed that the recombination rate between certain radicals was diffusion controlled, and could be affected by mixing strength. Their proposal, however, did not fit later observations (Pojman at al., 1992).

The last explanution was based on the large lateral concentration heterogeneity observed in a stirred tank (Menzinger and Jankowski, 1986, 1990). Rouff (1993) proposed that there existed many small cells in the tank containing low enough bromide ion concentration for igniting oscillations. The number of these excitable cells was assumed to decrease with increasing stirring rate. With this model, a reduction of oscillation period could be simulated when the stirring rate was lowered. Rouff's work is interesting but it is hard to prove experimentally due to its abstract nature.

In the present model, none of the mechanisms such as oxygen transfer, bromine loss, and difiusion mechanism are considered. From the calculated global concentration field shown in Fig. 9, the coexistence of many "nuclei" in the vessel, as proposed by RouI (1993), would generate a "CO" pattern, but not a simple oscillation pattern as discussed here.

Two results concerning the effects of nuclei are noticeable. First, each oscillation is ignited from the output zone and then spreads out to the whole vessel for $Q_{i}$ ranging from 50 to 1 . Actually, the output zone is the only excitable nucleus in the "SO" region. Second, when feed flow rate is reduced to 0.002 , the 


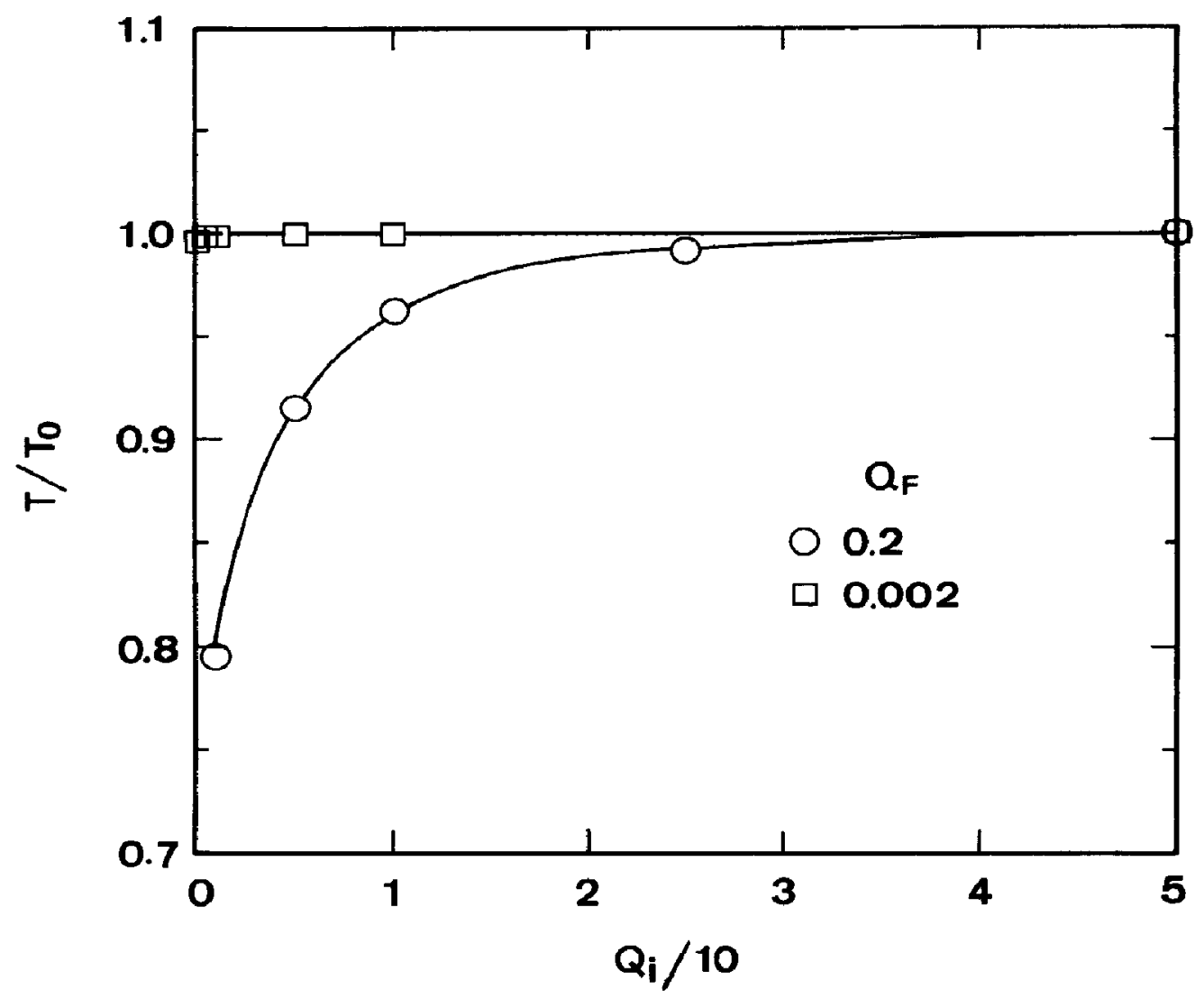

Fig. 8. Oscillation period vs internal flow rate. $K_{5}=200, f=0.9, N=10$.

oscillation periods become insensitive to the lowering of the stirring rate with the internal flow rate ranging from 50 to 0.1 .

These results demonstrate that the output zone is responsible for the observed lengthening of oscillations. $A$ better mixing and/or a lower feed flow rate would make the output zonc concentration morc easily averaged out by the other zones, which would prolong the time required for the output zone to reduce its bromide ion concentration to below critical value, and hence would lengthen the oscillation period. This is the new explanation proposed in this paper.

Although the possible roles played by oxygen transfer and/or bromine loss are not excluded in the present calculations, it is demonstrated that the lengthening oscillation period can be generated by the global fluid field.

Note that micromixing models also give a similar result (Chang, 1992), i.e. both macromixing and micromixing act in parallel. In studying other oscillating systems, such as the chlorite-iodide reaction (Fox and Villermaux, 1990b) or Brusselator (Villermaux, 1991), based on a macro/micromixing model, the oscillation period and occurrence were also found to depend on the mixing intensity. Since stronger stirring improves both macromixing and micromixing, the period of reduction should be more profound than those found in the present simulation.

\section{Apparent complex oscillations}

In the "CO" region, many local "nuclei" form stochastically all over the vessel. The wave fronts generated by these nuclei will propagate along the fluid flow and interact with each other, thus resulting in the complex oscillations observed. From the cross-correlation analysis, the zones in the present model can be viewed as groups of oscillators with strong interactions when they are close, or weak when they are far apart. Since, in many studies, complex dynamics can be generated by coupling several simple oscillators (Doumbouya and Schneider, 1993; Hauser and Schneider, 1994), it is therefore not surprising to find complex oscillation patterns in such a network of much higher complexity.

\section{Cessation of oscillations}

In some experimental studies, oscillations can be sustained under lower stirring rate but cease under high stirring rate (Sevcik and Adamcikova, 1989; Rouff, 1993). To simulate such a result, a case is examined as follows. 

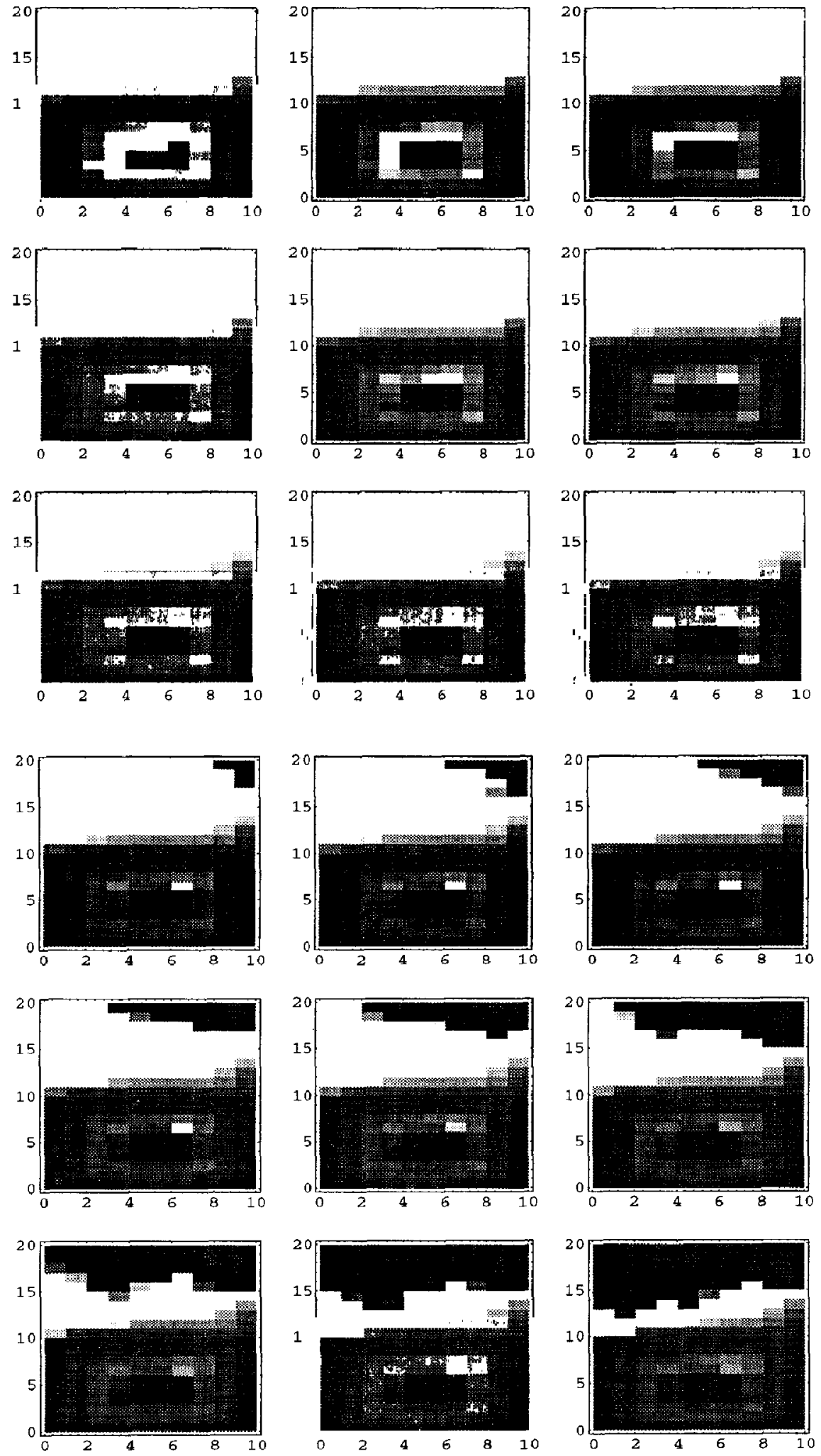

Fig. 9. Calculated global concentration field for component $X$. $K_{5}=200, f=0.9$, Parameter set no. 6 , $\tau=1000, t=72.1-72.9$. The sequence is from left to right, and from top to bottom with time interval 0.1 . 

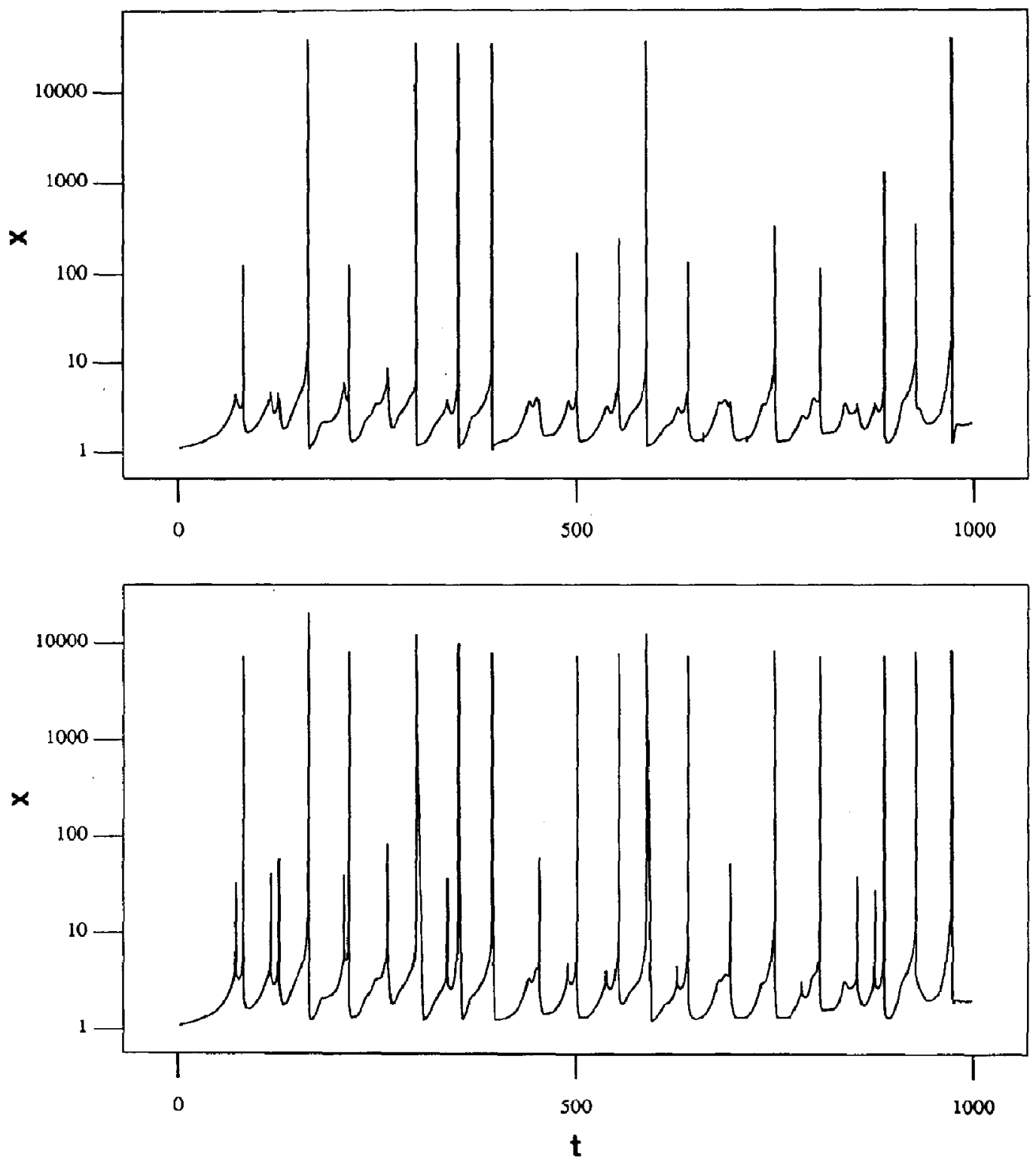

Fig. 10. Concentration for component $X$ vs time. $K_{5}=200, f=0.9$, Parameter set no. 6, $\tau=1000$. (a) Local concentration for zone $(5,2)$, and (b) average concentration for zone $(5,2)$ and its four nearest neighbors.

The disappearance of oscillation under high stirring rate has been interpreted as the enhancement of oxygen transfer and/or bromine loss (Sevcik and Adamcikova, 1988, 1989), or a decrease of excitable cell numbers (Rouff, 1993). For a first approximation, it is proposed that due to some mechanism, the bromine ion concentration for some local region, say zone $(1,1)$, is kept at a constant of 5,0 which is a high enough value to eliminate local oscillation. When the internal flow is low to medium (but still in the "SO" region), except for the zone just downstream to zone $(1,1)$, the oscillation patterns and the periods for other zones in the vessel are little affected by such a disturbance. However, when internal stirring rate is high, e.g. $Q_{i}=50$, due to the fast transport of the high concentration of bromide ions through the whole vessel, especially to the output zone, the oscillations can be quenched. Lowering the internal flow rate again, after an induction period, can re-establish oscillation. The calculated concentration sequences for zones $(1,2)$ and $(5,14)$ (solid curve) are demonstrated in Fig. 12. Such a result is qualitatively consistent with the experimental findings of Rouff (1993).

In a real stirred system, both macromixing and 


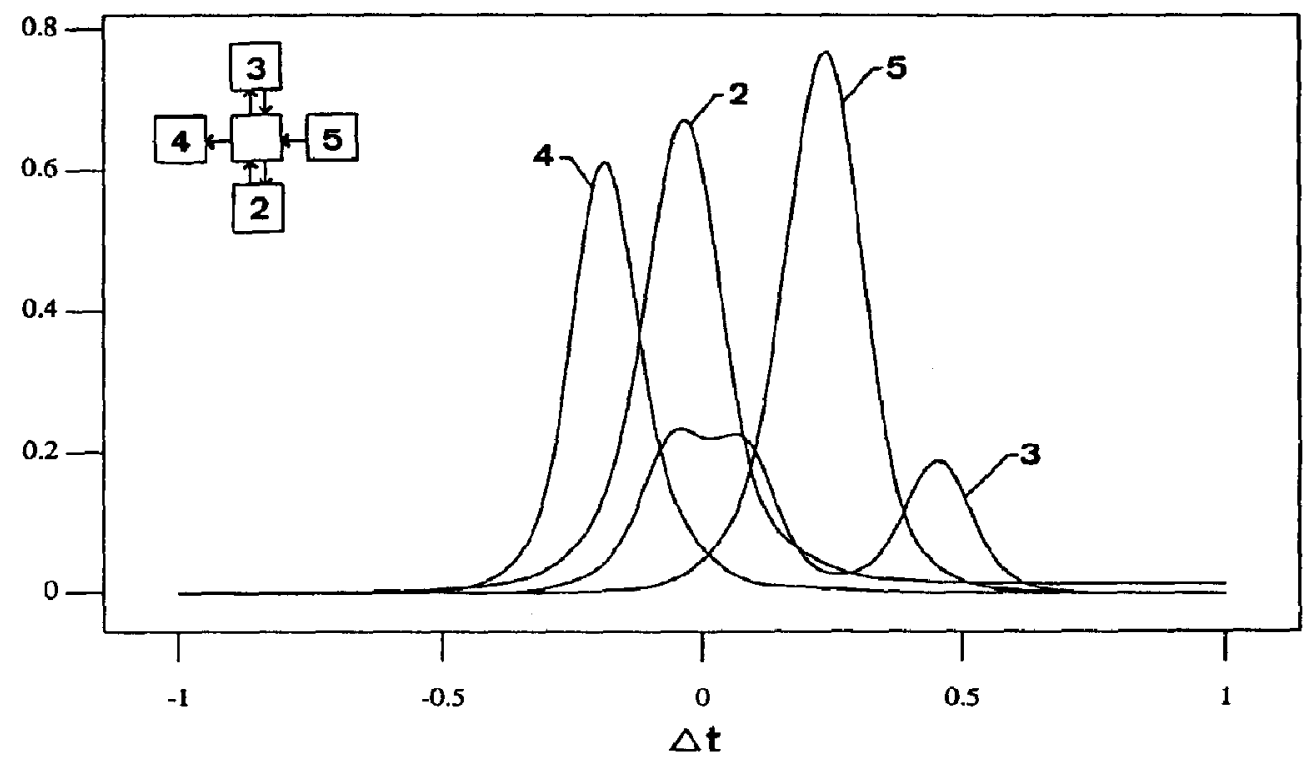

Fig. 11. Cross-correlation coefficients between zone $(5,2)$ with its four neighbors $(2-5)$. Low stirring rate (Parameter set no. 6).

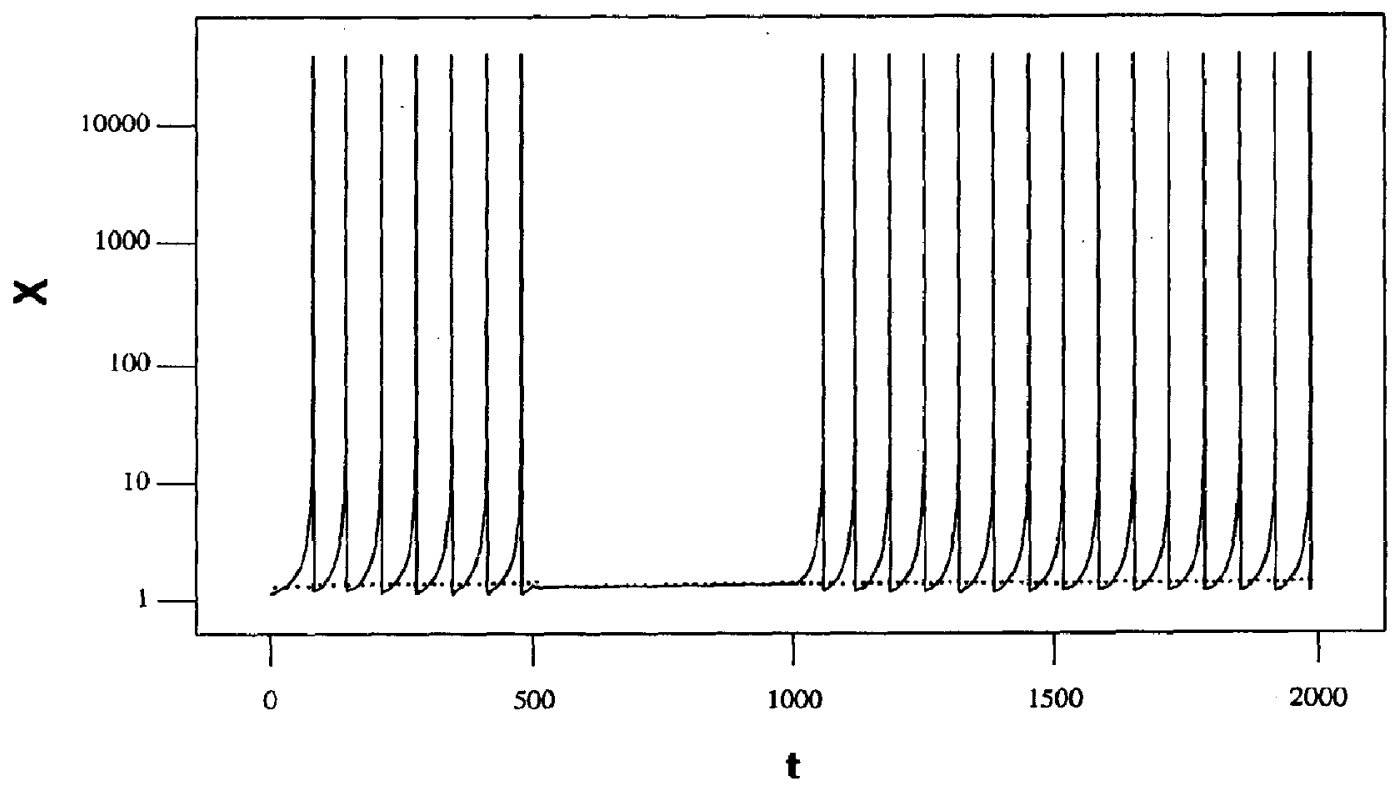

Fig. 12. Simulation for the cessation of oscillations. Concentration for component $Y$ for zone $(1,1)$ was kept as $5 . Q_{i}=1$ for $t$ in the range 0 to 500 , and changed to 50 when $t$ ranged from 500 to 1000 . After that, $Q_{i}$ was reduced back to $1 . K_{s}=200, f=0.9$.

micromixing exist. A mechanically well-mixed tank does not guarantee a truly uniform medium down to molecular scale, which is not involved in the present investigation. Besides, since the Oregonator is merely a simplified model [e.g. see Tyson (1981)], and since concentration fluctuation due to the internal flow between zones should be three-dimensional instead of the two-dimensional field employed here, the results shown in this work should be considered as preliminary. In spite of these limitations, this study clearly illustrated that the stirring rate can affect the oscillation periods, or even dramatically alter the system dynamics. Apparent complex dynamical behavior may be observed under incomplete mixing, though the original chemical kinetics possesses merely simple relaxation oscillations. 


\section{CONCLUSIONS}

Macromixing effects on the oscillating $\mathrm{BZ}$ reaction based on the global fluid field has been investigated. The three-variable irreversible Oregonator and the networks-of-zones model with $2 \times N \times N$ zones are chosen for describing the chemical kinetics and the flow field, respectively.

With a high stirring rate, all zones in the tank will oscillate synchronously with a period identical to the Orcgonator. An idcally wcll-mixed CSTR assumption can be adopted in such a case. When stirring rate is decreased, a simple oscillation pattern is found to be sustained locally, while a large lateral heterogeneity appears in the tank, and the oscillation period is decreased accordingly. It is proposed that the output cone acts as an excilable nucleus which determines the overall oscillations. When stirring rate is reduced further, many local zones can be excited as nuclei, and apparent complex oscillations can result.

\section{NOTATION}

$A=\left[\mathrm{BrO}_{3}^{-}\right]$

C dimensionless concentration

$f$ parameter in eq. (1e)

$K_{i}$ rate constant

$N$ parameter in networks-of-zones model

$P \quad=[\mathrm{HOBr}]$

$q$ parameter defined in eq. $(3 \mathrm{~g})$

$Q_{F}$ dimensionless feed flow rate

$Q_{i}$ dimensionless internal through flow rate

$r_{I}$ dimensionless reaction terms in eqs (5a)-(5c)

$s$ parameter defined in eq. ( $3 e$ )

$t$ dimensionless time

$t_{1}$ time, $\mathrm{s}$

$T$ dimensionless oscillation period

$T_{0}$ dimensionless oscillation period for a closed system

$V$ dimensionless volume

$w$ parameter defined in eq. (3f)

$x=\left[\mathrm{HBrO}_{2}\right]$

$X$ dimensionless concentration for $\mathrm{HBrO}_{2}$

$X_{0}$ dimensionless feed concentration for $\mathrm{HBrO}_{2}$

$y=\left[\mathrm{Br}^{-}\right]$

$Y$ dimensionless concentration for $\mathrm{Br}^{-1}$

$Y_{0}$ dimensionless feed concentration for $\mathrm{Br}^{-1}$

$z \quad 2\left[\mathrm{Ce}^{4+}\right]$

$Z$ dimensionless concentration for $\mathrm{Ce}^{+}$

$Z_{0}$ dimensionless feed concentration for $\mathrm{Ce}^{+4}$

\section{Greek letters}

$\alpha \quad$ fluctuation coefficient, $=0.2$

$\tau$ dimensionless residence time

\section{REFERENCES}

Argoul, F., Arneodo, A., Richetti, P, and Roux, J. C., 1987, From quasiperiodicity to chaos in the BelousovZhabotinskii reaction. I. Experiment. J. Chem. Phys. 86, 3325-3338.

Boissonade, J. and DeKepper, P., 1987, A comprehensive interpretation of mixing effects on stationary states and dynamical behavior of the bistable $\mathrm{ClO}_{2}^{-}-\mathrm{I}^{-}$reaction in a flow reactor. J. Chem. Phys. 87, 210-218.
Brucato, A. and Rizzuti, L., 1988, The application of the network-of-zones model to solid-liquid suspensions, in Proceedings of the 6th European Conference on Mixing, Pavia, Italy, pp. 273-280.

Chang, P. C., 1992, A study on chemical oscillation theories. Masters thesis, National Taiwan University, Taipei, Taiwan.

David, R., Muhr, H. and Villermaux, J., 1992, The yield of a consecutive-competitive reaction in a double jet semibatch reactor: comparison between experiments and a multizone mixing model. Chem. Engng Sci. 47, 2841-2846.

DeKepper, P. and Boissonade, J., 1981, Theoretical and experimental analysis of phase diagrams and related dynamic properties in the Belousov-Zhabotinskii system. J. Chem. Phys. 75, 189-195.

Doumbouya, S. I. and Schneider, F. W, 1993, Transition from quasiperiodicity to chaos in coupled oscillators. J. Phys. Chem. 97, 6945-6947.

Dutt, $\Lambda . K$. and Menzinger, M., 1990, Stirring and mixing effects on chemical instabilities: bistability of the $\mathrm{BrO}_{3}^{-}$/ $\mathrm{Br}^{-} / \mathrm{Ce}^{3+}$ system. J. Phys. Chem. 94, 4867-4870.

Dutt, A. K. and Menzinger, M., 1992, Effect of stirring and temperature on a Belousov-Zhabotinskii-like reaction in a batch reactor. $J$. Phys. Chem. 96, 8447-8449.

Dutt, A. K. and Muller, S. C., 1993, Effects of stirring and tcmpcrature on the Belousov-Zhabotinskii reaction in a CSTR. J. Phys. Chem. 97, 10059-10063.

Field, R. J. and Noyes, R. M., 1974, Oscillations in chemical systems. IV. Limit cycle behavior in a model of a real chemical reaction. J. Phys. Chem. 60, 1877-1884.

Field, R. J., Koros, E. and Noyes, R. M., 1972, Oscillations in chemical systems. II. Through analysis of temporal oscillation in the bromate-cerium-malonic acid system. J. Am. Chem. Soc, 94, 8649-8664.

Fox, R. O. and Villermaux, J., 1990a, Unsteady-state IEM model: numerical simulation and multiple-scale perturbation analysis near perfect-micromixing limit. Chem. Engng Sci. 45, 373-386.

Fox, R. O. and Villermaux, J., 1990b, Micromixing effects in the $\mathrm{ClO}_{2}^{-}+\mathrm{I}^{-}$reaction: perturbation analysis and numerical simulation of the unsteady-state IEM model. Chem. Engng Sci. 45, 2857-2876.

Ganapathisubramanian, N., 1991, Tristability in the iodate/ As(III) chemical system arising from a model of stirring and mixing effects. J. Chem. Phys. 95, 3005-3008.

Gyorgyi, L. and Field, R. I., 1989. Aperiodicity resulting from two-cycle coupling in the Bclousov-Zhabotinskii reaction. 2. Modeling of the effect of dead spaces at the input ports of a continuous-flow stirred tank reactor. J. Phys. Chem. 93, $2865-2869$.

Gyorgyi, L. and Field, R. J., 1992, Simulation of the effect of stirring rate on bistability in the $\mathrm{BrO}_{3}^{-}-\mathrm{Ce}$ (III) $-\mathrm{Br}^{-}$ CSTR reaction. J. Phys. Chem. 96, 1220-1224.

Hauscr, M. J. B. and Schneider, F. W., 1994, Coupled chaotic states and apparent noise in experiment and model. J. Chem. Phys. 100, 1058-1065.

Hauser, M. J. B., Lebender, D. and Schneider, F. W., 1992, Stirring sense discriminates between stationary and oscillatory states. J. Chem. Phys. 97, 2163-2165.

Hannon, L. and Horsthemke, W. 1987, Stirring effects and bistability in the iodate-arsenous acid reaction: premixed vs segregated flows. J. Chem. Phys. 86, 140-143.

Horsthemke, W. and Hannon, L. 1984, Nonequilibrium chemical instabilities in continuous flow stirred tank reactors: the effect of stirring. J. Chem. Phys. 81, 4363-4368.

Kumpinsky, E. and Epstein, I. R., 1985, A model for stirring effects on transitions in bistable chemical systems. J. Chem. Phys. 82, 53-57,

Knysh, P. and Mann, R., 1984, Utility of networks of interconnected backmixed zones to represent mixing in a closed stirred vessel. Inst. Chem. Engng Symp. Ser. 89, $127-145$. 
Levenspiel, O., 1972, Chemical Reaction Engineering. John Wiley \& Sons, New York.

Lindberg, D., Turner, J. S. and Barkley, D., 1990, Chaos in the Showalter-Noyes-Bar-Eli model of the BelousovZhabotinskii reaction. $J$. Chem. Phys. 92, 3238-3239.

Lopez-Tomas, L. and Sagues, F., 1991, New features of stirring sensitivities of the Belousov-Zhabotinskii reaction. J. Phys. Chem. 95, 701-705.

Luo, Y. and Epstein, I. R., 1986, Stirring and premixing effects in the oscillatory chlorite-iodide reaction. J. Chem. Phys. 85, 5733-5740.

Mann, R., 1986, Gas-liquid stirred vessel mixers: towards a unified theory based on the network-of-zones. Chem. Engng Res. Des. 64, 23-34.

Mann, R. and El-Hamouz, A., 1991, Effect of macromixing on a competitive/consecutive reaction in a semi-batch stirred reactor: Paul's iodination experiments interpreted by networks-of-zones. Proceedings of the 7 th European Conference on Mixing, Brussels, Belgium, pp. 1-8.

Mann, R, and Hackett, L. A., 1988, Fundamentals of gas-liquid mixing in a stirred vessel: an analysis using networks of backmixed zones. Proceedings of the 6th European Conference on Mixing, Pavia, Italy, pp. 321-328.

Mann, R. and Mavros, P., 1982, Analysis of unsteady tracer dispersion and mixing in a stirred vessel using interconnected networks of ideal flow zones. Proceedings of the 4th European Conference on Mixing, Nordwijkerhout, pp. $35-47$.

Mann, R., Knysh, P., Rasekoala, E. A. and Didari, M., 1987, Mixing in a closed stirred vessel: use of networks-of-zones to interpret mixing curves acquired by fibre-optic photometry. Inst. Chem. Engng Symp. Ser. 108, 49-62.

Menzinger, M. and Dutt, A. K., 1990, The myth of the well-stirred CSTR in chemical instability experiments: the chlorite/iodide reaction. J. Phys. Chem. 94, 4510-4514.

Menzinger, M. and Giraudi, A., 1987, Stirring effects in chemical instabilities: heterogeneity induced oscillations in the $\mathrm{ClO}_{2}^{-}+\mathrm{I}^{-}$reaction. J. Phys. Chem. 91, $4391-4393$.

Menzinger, M. and Jankowski, P., 1986, Heterogeneities and stirring effects in the Belousov-Zhabotinsky reaction. J. Phys. Chem. 90, 1217-1219.

Menzinger, M. and Jankowski, P., 1990, Concentration fluctuations and stirring effects in the BelousovZhabotinsky reaction. J. Phys. Chem. 94, 4123-4126.

Menzinger, M., Boukalouch, M., DeKepper, P., Boissonade, J., Roux, J. C. and Saadaoui, H. 1986, Dynamical consequences of nonideal mixing in continuously stirred tank reactor studies. $J$. Phys. Chem. 90, 313-315.

Nauman, E. B. and Buffham, B. A., 1983, Mixing in Continuous Flow Systems. John Wiley \& Sons, New York.

Nienow, A. W., Drain, S. M. and Boyes, A. P., 1992, A new pair of reactions to characterize imperfect macro-mixing and partial segregation in a stirred semi-batch reactor. Chem. Engng Sci. 47, 2825-2830.

Noszticzius, Z, Bodnar, Z, Garamszergi, L. and Wittmann, M., 1991, Hydrodynamic turbulence and diffusion-controlled reactions. Simulation of the effect of stirring on the oscillating Belousov-Zhabotinsky reaction with the radicalator model. J. Phys. Chem. 95, 6575-6580.

Ochiai, E.-I. and Menzinger, M., 1990, Chemical instabilities: a spectroscopic study of spatial inhomogeneities in the $\mathrm{ClO}_{2}^{-} / \mathrm{I}^{-}$reaction in a continuously stirred tank rcactor. J. Phys. Chem. 94, 8866-8868.

Orban, M. and Epstein, I. R., 1992, A new type of oxyhalogen oscillator: the bromite-iodide reaction in a continuous flow reactor. J. Am. Chem. Sac. 114, 1252-1256.

Pojman, J. A., Dedeaux, H. and Fortenberry, D., 1992, Surface-induced stirring effects in the Mn-catalyzed Belousov-Zhabotinskii reaction with a mixed hypophosphite/acetone substract in a batch reactor. $J$. Phys. Chem. 96, 7331-7333.

Puhl, A. and Nicolis, G., 1986, Micromixing and multiple steady state transitions in a CSTR. Chem. Engng Sci. 41, 3111-3117.

Puhl, A. and Nicolis, G., 1987, Normal form analysis of multiple bifurcations in incompletely mixed chemical reactors. J. Chem. Phys. 87, 1070-1078.

Razon, L. F. and Schmitz, R. A., 1987, Multiplicities and instabilities in chemical reacting systems-A review. Chem. Engng. Sci. 42, 1005-1047.

Roux, J. C., DeKepper, P. and Boissonade, J., 1983, Experimental evidence of nucleation induced transition in a bistable chemical system. Phys. Lett. 97A, 168-170.

Ruoff, P., 1993, Excitations induced by fluctuations: an explanation of stirring effects and chaos in closed anaerobic classical Belousov-Zhabotinsky systems. J. Phys. Chem. 97, 6405-6411.

Sevcik, P. and Adamcikova, I., 1988, Stirring rate effect in the Belousov-Zhabotinsky reaction. Chem. Phys. Lett. 146, 419-421.

Sevcik, P. and Adamcikova, I., 1989, Stirring rate effect in the closed, batch Belousov-Zhabotinsky system with oxalic acid. J. Chem. Phys. 91, 1012-1014.

Showalter, K., Noyes, R. M. and Bar-Eli, K., 1978, A modified Oregonator model exhibiting complicated limit cycle behavior in a flow system. J. Chem. Phys. 69, 2514-2524.

Tyson, J. J., 1977, Analytical representation of oscillations, excitability, and traveling waves in a realistic model of the Belousov-Zhabotinskii reaction. J. Chem. Phys. 66, 905-915.

Tyson, J. J., 1981, On scaling the Oregonator equations, in Nonlinear Phenomena in Chemical dynamics (Edited by C. Vidal and A. Pacault), pp. 222-227.

Vanag, V. K. and Alfimov, M. V., 1993, Effects of stirring on photoinduced phase transition in a batch-mode Briggs-Rauscher Reaction. J. Phys. Chem. 97, 1884-1890.

Villermaux, J., 1991, Mixing effects on complex chemical reactions in a stirred reactor. Rev, Chem. Engng, 7, 51-108.

Voit, H. and Mersmann, A. B., 1988, Calculation of mixing times from the field of mean and fluctuating velocity profiles. Proceedings of the 6 th European Conference on Mixing, Pavia, Italy, pp. 15-21.

Wang, Y.-D. and Mann, R., 1992, Partial segregation in stirred batch reactors: effect of scale-up on the yield of a pair of competing reactions. Trans. Inst. Chem. Engrs 70, 282-290. 